\title{
PROPUESTA DE CUESTIONARIO PARA MEDIR EL NIVEL DE CONOCIMIENTO SOBRE LAS FINANZAS PERSONALES DE JÓVENES UNIVERSITARIOS
}

\section{PROPOSAL OF A QUESTIONNAIRE TO MEASURE THE KNOWLEDGE LEVEL OF PERSONAL FINANCES IN UNIVERSITY STUDENTS}

\begin{abstract}
Angela Araceli Estrada Martínez; Paula Arévalo Guzmán; Shirley Jenine Moya Pantoja; Claudia Maria José Aranda Magallanes; Stefanía Jeniffer Rivera Sánchez.

Estudiantes de la Universidad Ricardo Palma

Facultad de Ciencias Económicas y Empresariales.

Escuela Profesional de Administración de Negocios Globales
\end{abstract}

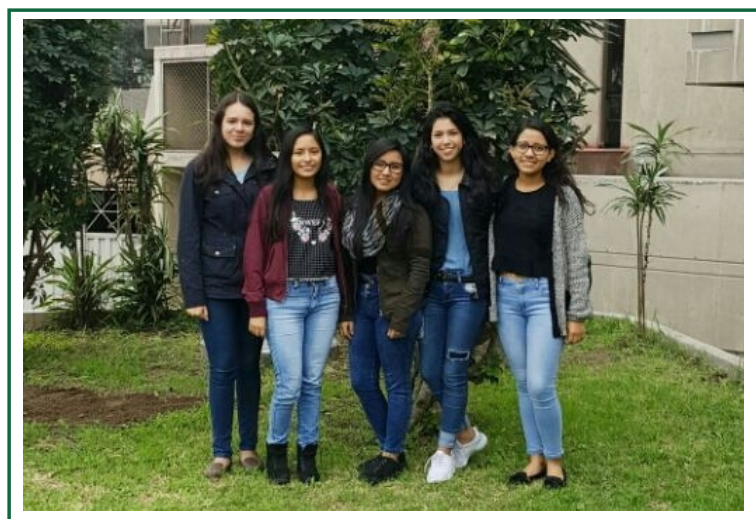

Recepción: 2017-03-29 / Aceptación: 2017-05-05

\section{RESUMEN}

cuestionario para medir el nivel de conocimientos El objetivo de esta investigación es proponer un cuestionario para medir el nivel de conocimientos
sobre finanzas personales en jóvenes universitarios estudiantes de carreras de ciencias económicas. Para realizar este estudio, se extrajo información de tesis, artículos y libros sobre las finanzas personales; en base a lo cual se elaboró una primera aproximación teórica. Se concluye que es necesario que los estudiantes universitarios comprendan la importancia de conocer sobre las finanzas personales, el ahorro y los presupuestos personales ya que son esenciales para tomar buenas decisiones aprovechando óptimamente sus recursos económicos.

Palabras clave: Finanzas personales, ahorro, presupuestos.

\section{ABSTRACT}

The objective of this research is to propose a questionnaire to measure the level of knowledge that university students of economics have about personal finances. To do the research, thesis, articles and books about topics related with personal finances have been used; based on which a first theoretical approach has been made. It is concluded that university students need to understand the importance to know about personal finances, saving and personal budgets, since they are essential to make good decisions taking ideal advantage of their economic resourses. Finally, it is necessary for university students to understand how important is to know personal finances, savings and budgets to make good decisions using their financial resources correctly.

Keywords: Personal finances, savings, budget. 


\section{INTRODUCCIÓN}

El crecimiento económico permite alcanzar objetivos importantes para las personas y las sociedades, como por ejemplo, la reducción de la pobreza, el empleo productivo, la educación, la salud y las oportunidades para ser creativos. Sin crecimiento económico, es muy complicado alcanzar plenamente esos objetivos (Banco Mundial, 2011).

El crecimiento económico genera la necesidad de que las personas sepan cómo manejar sus finanzas personales y beneficiarse de los mercados financieros más complejos. En este sentido, las iniciativas de educación financiera pueden convertirse en un complemento importante de los procesos de inclusión financiera y las medidas de reducción de la pobreza (García, Grifoni, López y Mejía, 2013).

Según Navickas, Tadas y Krajnakova (2013) las finanzas personales son, de manera simplificada, todas las decisiones financieras y actividades que una persona puede hacer; incluyendo los ahorros, ingresos, gastos, y todo lo que requiera dinero.

El Banco Mundial (2011) indica que América Latina apunta a crear políticas dirigidas a obtener un mayor acceso a la educación y la salud, una mejor infraestructura física, un mejor entorno para el desarrollo del sector privado, apertura comercial, liberalización de mercados, etc., lo cual ha tenido efectos positivos directos tanto sobre el crecimiento económico como sobre la disminución de la desigualdad y la reducción de la pobreza. El Banco mundial (2011) también sustenta que pese a la crisis económica global del 2009, el Perú ha logrado crecer del en el último 2004 al 2009 a una media del $7 \%$ generando suficientes empleos en el sector formal para absorber las tendencias demográficas y reducir la informalidad.

El ahorro es el proceso mediante el cual una economía reserva parte de su producto y lo utiliza para generar ingresos en el futuro. El nivel de ahorro que prevalece en un país está determinado por la elección de consumo y ahorro, lo que contribuye a la determinación de la tasa de crecimiento de la economía. Pero la influencia del ahorro sobre tal indicador se establece mediante la inversión (Tomala y Gonzalez, 2009).

El ahorro de las personas que tienen bajos ingresos está destinado principalmente para educación; para aquellos que tienen mayores niveles de ingreso su ahorro está destinado principalmente como reserva para el futuro o para solventar situaciones imprevistas (Nima, 2009).

Meli y Bruzzone (2006) refieren que actualmente en el mercado podemos encontrar diversos instrumentos de ahorro, los que se deben estudiar cuidadosamente a fin de optar por uno que nos genere tranquilidad, permitiendo mantener en el tiempo el plan propuesto, así como proporcionando la confianza de no perder o ver disminuido nuestro capital.

Según Denegri, Martínez y Etchebarne (2007), en la mayoría de los jóvenes de 18 años se observan algunas lagunas conceptuales reflejadas especialmente en la dificultad para comprender términos relacionados con las finanzas personales. 
recibida en el hogar, podemos anticipar que estos jóvenes tienen altas probabilidades de convertirse en usuarios habituales del crédito con alta vulnerabilidad a sobre endeudarse. De esta forma se evidencia la importancia de una adecuada socialización económica y de la inserción de la educación económica en el currículum formativo que le permita al sujeto adquirir creencias, valores, actitudes y conductas adecuadas en relación al consumo (Denegri, Cabezas, Páez, Sanhueza, Vargas, Zapata y Sepúlveda, 2010).

Los objetivos son:

Estructurar la base teórica sobre finanzas personales de jóvenes universitarios.

Proponer un cuestionario que mida el nivel de conocimientos sobre finanzas personales de jóvenes universitarios de carreras de ciencias económicas.

\section{MATERIAL Y MÉTODOS}

Se estructuró la base teórica a partir del análisis de tesis, artículos y libros. Además se realizó una entrevista a un profesional con experiencia laboral en el Sistema financiero.

\section{RESULTADOS}

En un estudio realizado a jóvenes chilenos, se demostró que gran parte de ellos tienen un escaso conocimiento sobre el uso adecuado de sus finanzas y por ello no saben cómo ahorrar y en qué lugar hacerlo. Es por eso que la Alfabetización. Económica es fundamental, ya que nos ayuda a comprender el mundo económico y mejorar nuestra toma de decisión personal al momento de enfrentar un problema económico de la vida diaria. Así mismo, una adecuada Alfabetización Financiera desarrolla la habilidad para balancear ingresos y gastos, mantener una cuenta corriente saludable, preparar y administrar un presupuesto, desarrollar estrategias de ahorro y manejar en forma eficiente el uso del crédito, tanto cotidianamente, como en una proyección hacia el futuro (Denegri, Martínez y Etchebarne, 2007).

Por lo tanto, gracias a una alfabetización financiera podríamos poner en práctica las finanzas personales adecuadas y convenientes según el interés propio, estos conocimientos financieros se deben fomentar desde las primeras etapas educativas del ser humano. Tomamos a los jóvenes como centro de análisis en esta propuesta de cuestionario, ya que los errores financieros en un futuro pueden ser difíciles de corregir.

Podemos definir a las finanzas personales como toda decisión financiera y actividades que incluyen el presupuesto de ingresos y gastos de los hogares, ahorros, inversiones, hipotecas, seguros y todas las demás decisiones que requieren dinero, estableciendo objetivos a corto plazo y a largo plazo. Una manera de tener habilidad en la administración de las finanzas, es poniendo en práctica las finanzas personales, realizando un presupuesto en el cual se estimen todos los ingresos y gastos para cada periodo establecido, sea mensual, trimestral, anual, etc.

La organización Freedom from Hunger sostiene que cuando los jóvenes empiezan a ahorrar 
desde temprana edad, aumentan su potencial de desarrollar un hábito de ahorro que pueden seguir practicando en su vida adulta, fortaleciendo sus capacidades financieras a medida que empiezan a afrontar mayores responsabilidades sociales y económicas. Inculcar un interés ayudará a crear conciencia sobre la importancia de manejar responsablemente el dinero y que se plantee metas que pueda lograr mediante el ahorro (Ramírez, R. y Fleischer, L., 2013).

Un estudio sobre jóvenes chilenos reporta que no tienen conciencia suficiente de la importancia del ahorro. Lo cual se refleja en las dificultades que presentan para entender términos de finanzas (Denegri, Martínez y Etchebarne, 2007).

Esa edad es crucial porque algunos jóvenes empiezan a percibir ingresos y existen un interés por gastarlo en diversas actividades.

Un ser humano asume actitudes o toma posición respecto a distintos objetos y personas.

Por ejemplo, la actitud hacia el dinero, puede influir en el comportamiento, uno de los comportamientos de las personas que tal vez está muy ligado al dinero es el comportamiento del consumidor el cual teóricamente se limita a su capacidad de compra. Lo mismo sucede con los jóvenes que están entrando recién al mundo económico y de consumo, sin una formación adecuada para poder afrontar toda esta información que tiene como objetivo hacer de las personas consumidores potenciales. En este afán poco se han ocupado de los consumidores como personas, es por eso que han surgido una serie de problemas relacionados con el consumo como por ejemplo la compra impulsiva y deudores. (Santa María y Gómez, 2005, p.5).
Todas las personas tenemos objetivos, metas y necesidades, y para ello es necesario analizar, planificar y prever los recursos que necesitamos para alcanzar dichos propósitos. Las finanzas personales no son ajenas a los diversos ámbitos de la vida; por el contrario, manejar responsablemente nuestras finanzas personales nos permite distribuir mejor nuestros recursos y así poder realizar óptimamente las actividades deseadas. Por ejemplo; los jóvenes universitarios no solo tienen gastos académicos, también realizan gastos para adquirir ciertos objetos personales o participar reuniones y salidas con amigos en su tiempo libre; algunos jóvenes ahorran porque tienen planes a mediano o largo plazo claramente establecidos. Para todas esas actividades y muchas otras más, que son comunes en los jóvenes, es necesario tener orden en las finanzas personales y una herramienta muy útil es realizar un presupuesto personal.

La palabra presupuesto proviene de las siguientes raíces latina: "pre" que significa delante de y "supuesto" que significa hecho, dado por cierto. Entonces la palabra presupuesto significa antes de los hechos.

Para Olmedo (2009) el presupuesto es una herramienta que nos permite identificar los recursos económicos y los gastos que tenemos, la importancia del ahorro y del consumo futuro.

Además el autor sostiene que tener un presupuesto nos permite contar con un marco de referencia para tomar decisiones financieras acertadas, que permitan alcanzar sus metas y sueños, en el contexto de una adecuada calidad de vida personal.

Burbano y Ortiz (1995) sostiene que los pre- 
supuestos son un medio para maximizar el rendimiento de nuestros recursos. Los autores también mencionan que hay casos en los que algunas personas, incluidos jóvenes, que afirman haber tenido unas buenas finanzas personales sin haber elaborado presupuestos; pero en realidad todas las decisiones que han tomado han sido previamente analizadas. Entonces, en esencia las personas presupuestan pero de manera abstracta y muchas veces sin estar conscientes de ello.

Los presupuestos se caracterizan por:

a) Abarcar un periodo de tiempo específico, similar a las agendas donde anotamos nuestras reuniones, tareas, etc., donde las fechas están indicadas y la planificación es en periodos de tiempo determinados. Normalmente, los presupuestos personales se elaboran en periodos mensuales pero como se trata de un presupuesto personal para estudiantes universitarios, y ya que sus gastos no suelen ser en periodos mensuales sino semanales, elaborar un presupuesto personal semanal también es una opción recomendable para ellos.

b) Los registros se realizan en términos monetarios. Al registrar los ingresos, gastos, ahorros y deudas en el presupuesto, esto se hace numéricamente, por ello es recomendable ser ordenado al registrar cada monto y preferiblemente hacerlo en una sola moneda para evitar confusiones.

c) Se basan en registros pasados de ingresos, gastos, ahorros y deudas que permiten proyectar la cantidad de recursos financieros que tenemos para hacer frente a nuestras responsabilidades y objetivos.

d) Son flexibles ya que como se trata de proyecciones, siempre las circunstancias no son como las que se habían planeado, por este motivo es recomendable tener un monto del presupuesto destinado a contingencias.

Habiendo explicado la importancia de adquirir conocimientos sobre las finanzas personales, proponemos un cuestionario que permita medir el nivel de conocimiento sobre las finanzas personales en jóvenes universitarios peruanos, específicamente en los que estudian carreras de ciencias económicas.

\section{DISCUSIÓN}

La aproximación teórica lograda corrobora lo propuesto por Denegri, Martínez y Etchebarne (2007) pues todo universitario debería adquirir conocimiento sobre sus finanzas y para eso es importante la alfabetización financiera durante la educación, la cual actúa como base y a la vez permite tener ciertas habilidades al momento de realizar un presupuesto, balancear ingresos y gastos así como también desarrollar estrategias para poder ahorrar. Además, se coincide con lo mencionado por los autores Navickas, Gudaitis y Krajnakova (2013) en referencia a que la alfabetización financiera es clave para poder entender y poner en práctica de la mejor manera las finanzas personales, puesto que nos facilitara el entendimento y aplicación de esta.

Por otra parte, se concuerda con Gómez (2015), quien sostiene que los jóvenes creen que no pueden ahorrar por no tener ingresos suficien- 


\section{Tabla 1}

Indicadores e items de cuestionario para medir el nivel de conocimientos sobre finanzas personales de jóvenes universitarios.

CONCEPTUAL

INDICADORES

Estudiante posee base sobre nociones financieras que le ayuda a comprender el mundo económico.
ITEMS

En caso que tenga dinero ahorrado, ¿En qué lugar le gustaría guardarlo?.
a) Alcancía
b) Entidad Financiera
c) No responde

Decisión Financiera (Navickas, Gudaitis y Krajnakova, 2013).
Estudiante tiene capacidad de decisión sobre su dinero.
En caso que tenga dinero sobrante después de realizar sus pagos. ¿Qué haría con dicho dinero excedente?
a) Ahorra
b) Invierte
c) Gasta
d) No responde

La importancia del conocimiento sobre las finanzas personales (Ramírez y Fleischer, 2013).
El estudiante considera importante tener conocimientos sobre finanzas personales.
¿Usted considera importante tener conocimientos sobre fianzas personales?

a) Muy importante

b) Poco importante

c) Nada importante
¿Usted tiene el hábito de ahorrar?
a) A veces ahorro, pero luego lo gasto.
b) Sí, siempre que tengo un ingreso, ahorro una parte.
b) No ahorro.

Hábito de ahorro (Meli

El estudiante posee hábito hacia el ahorro.
Si usted ahorra ¿Qué meta le gustaría lograr?
a) A veces ahorro, pero luego lo gasto.
b) Sí, siempre que tengo un ingreso, ahorro una parte.
c) No ahorro. 
Manejo responsable del ahorro de dinero (Nima, 2009)
El estudiante maneja responsablemente el dinero

En el caso de que tenga dinero sobrante después de realizar sus pagos. ¿Qué haría con dicho dinero excedente?
a) Ahorra
b) Invierte
c) Gasta

Si usted ahorra ¿Qué meta le gustaría lograr?

Metas a lograr mediante el ahorro (Gómez, 2015).......
Se plantea metas

a lograr mediante el ahorro. a) Ir de vacaciones

b )Invertir para generar más ingresos.

c) Comprarme un carro moderno

d) Comprarme algo que me guste

Indique cuál de las siguientes opciones debe registrarse en

Conocimientos para elaborar un presupuesto (Burbano y

Ortiz, 1995)
Estudiante tiene conocimientos de cómo elaborar un presupuesto un presupuesto:
a) Ingresos
b) Gastos
c) Deudas
d) Ahorros
e) Todas las anteriores

Fuente: Elaboración propia

tes al estar en una edad en la que son dependientes de sus padres, por lo que al momento de plantearse una meta se dan cuenta de la importancia del ahorro para lograrla

Una manera de incentivar el ahorro es comenzar planteándose pequeñas metas de modo que no parezca complicado. Para ello, tener una adecuada Alfabetización Financiera permitirá a los jóvenes adquirir la habilidad para balancear ingresos y gastos, mantener una cuenta corriente saludable, preparar y administrar un presupuesto, desarrollar estrategias de ahorro y ma- nejar en forma eficiente el uso del crédito, tanto cotidianamente, como en una proyección hacia el futuro (Denegri, Martínez y Etchebarne,2007). La importancia de presupuestar nuestro dinero nos permitirá llevar un registro más ordenado de las finanzas personales lo cual se reflejará en la mejora de futuras decisiones financieras.

Como mencionan Burbano y Ortiz (1995) las personas pueden hacer sus presupuestos sin estar conscientes de ello, entonces si somos capaces de planificarlo y plasmarlo en físico no es complicado y basta con un pequeño asesoramiento para hacerlo de la manera más óptima 
Finalmente, la importancia del conocimiento sobre las finanzas personales, el ahorro y los presupuestos personales son esenciales para tomar buenas decisiones aprovechando óptimamente los recursos económicos que posee un estudiante, los cuales generalmente son proporcionados por sus padres. Por tanto, consideramos que todos estos conocimientos deben incluirse en la formación de las personas desde temprana edad de modo que pueda convertirse en un hábito. Cabe resaltar que la adopción de estas prácticas sobre finanzas personales está predominantemente influenciada por factores psicológicos y culturales, pues aun teniendo los conocimientos necesarios, pesan más al momento de tomar decisiones respecto a las finanzas personales.

\section{REFERENCIAS}

Banco Mundial. (2011). Perú en el umbral de una nueva era. Lima: Banco Mundial.

Burbano, J.E., Ortiz, A. (1995). Presupuestos enfoque moderno de planeación y control de recursos. (2da ed.). Bogotá: McGraw-Hill.

Denegri, M., Cabezas, D., Páez, A., Sanhueza, O., Vargas, M., Zapata, L. \& Sepúlveda, J. (2010). Actitudes hacia el endeudamiento en adolescentes de educación municipal y particular-subvencionada de la ciudad de Temuco. Revista de Educación y Humanidades, 1(2), pp. 46-63.

Denegri, M., Martínez, G. \& Etchebarne, S. (2007). La comprensión del funcionamiento bancario en adolescentes chilenos: un estudio de Psicología Económica. Interdisciplinaria, 2(24), pp. 137-159.
García, N., Grifoni, A., López, J. C., \& Mejía, D. (2013). La educación financiera en América Latina y el Caribe. Situación actual y perspectivas. Serie Políticas Públicas y Transformación Productiva. Caracas: Banco de desarrollo de América Latina.

Gómez, C. (2015). Propuesta para Incrementar la Cultura Financiera en Estudiantes Universitarios en la Ciudad de San Luis Potosí. (Tesis para optar el grado de Maestro en Administración con Énfasis en Negocios). Universidad Autónoma de San Luis Potosí, San Luis de Potosí, México.

Meli, J. \& Bruzzone, P. (2006). El dinero y E ahorro.I Recuperado de http://www.economicas.unsa. edu.ar/afinan/informacion_general/book/libro _dinero_y_ahorro.pdf

Navickas, M., Gudaitis, T., Krajnakova, E.(2013). Influence of financial literacy on management of personal finances in a young household. Business: Theory and Practice. 15 (1), pp. $32-40$

Nima, G. (2009). Los determinantes del ahorro. Obtenido de http://revistas.up.edu.pe/index. php/apuntes/article/view/269/260

Olmedo, L. (2009). Las finanzas personales. Revista Escuela de Administración de Negocios, 65, pp.123 -144. Recuperado de http:// www.redalyc.org/articulo.oa?id=20612980007

Ramírez, R. \& Fleischer, V. (2013). Ahorrando Juntos: Enfoque en grupos para promover el ahorro entre jóvenes. Freedom from Hunger. 
Recuperado el 22-06-17 desde https://www. freedomfromhunger.org/sites/default/files/do cuments/SavingTogether_Spa.pdf

Santa María, C. \& Gómez, J. (2005). Actitudes hacia el dinero en jóvenes de 18 a 23 años. Ajayu Órgano de Difusión Científica del Departamento de Psicología UCBSP, 3 (1), 86-107.
Tomala, J. \& Gonzalez, M. (2009).Ahorro y crecimiento económico: evidencia empírica de causalidad para el período 1970-2002. Escuela Superior Politécnica del Litoral, Guayaquil, Ecuador. Recuperado desde http://www.dspace. espol.edu.ec/handle/123456789/516

\section{Correo para correspondencia} angela0021.es@gmail.com 